\title{
Evaluation of liquid culture media to support growth of Mobiluncus species
}

\author{
A. W. TAYLOR-ROBINSON* and D. TAYLOR-ROBINSON $\dagger$ \\ * School of Biology, University of Leeds, Leeds LS2 9JT and †Imperial College School of Medicine, St Mary's \\ Hospital, London W2 $1 \mathrm{NY}$
}

\begin{abstract}
Mobiluncus curtisii and $M$. mulieris are anaerobic, gram-negative, motile curved rods isolated commonly from the vagina of women with bacterial vaginosis. Hitherto, there has been difficulty in isolating and growing these bacteria and little attention has been paid to growth in liquid media. Reasons for establishing the means of attaining optimal growth in such media include production of antigens for diagnostic and immunological studies and production of the soluble cytotoxin. In this study the efficacy of 12 liquid culture media in supporting growth was examined. M. mulieris (strain A198) multiplied $\geqslant 10$-fold in only five media - Schaedler broth, Columbia blood broth (CBB), peptonestarch-dextrose (PSD) broth, brain-heart infusion plus arginine and spent tissue-culture medium. Similarly, M. curtisii (strain A98) multiplied $\geqslant 10$-fold in only three media Schaedler broth, CBB and PSD. Some strains of both bacterial species grew very poorly or not at all, in all the media tested. With an inoculum of $\geqslant 10^{5} / \mathrm{ml}$, CBB, or PSD plus $10 \%$ horse serum, supported the growth of some strains of both bacterial species to $10^{9}$ organisms/ml within $48 \mathrm{~h}$, and viable bacteria persisted longer in some media (e.g., CBB) than in others. While variation in growth of Mobiluncus spp. may occur between one laboratory and another, these observations provide the basis for optimisation of a universal liquid culture medium that should facilitate production of antigens and cytotoxin.
\end{abstract}

\section{Introduction}

Mobiluncus curtisii and M. mulieris are anaerobic, gram-negative motile curved rods isolated from the vagina, particularly of women with bacterial vaginosis (BV) [1]. Microscopic detection of motile curved rods in a wet smear has been used as a criterion for the presence of Mobiluncus spp. in a vaginal specimen [2], but this procedure may also detect other motile bacteria unrelated to the curved rods. Therefore, it was important to find a means of isolating Mobiluncus spp. selectively in primary culture, a difficult task because the anaerobic flora of the vagina is profuse ( $10^{12}$ bacteria/g of secretion). Approaches to this objective have concentrated on attempts to inhibit the growth of other more rapidly growing bacteria present in vaginal specimens by using antibiotics and other agents in solid media [3-5]. This may be successful but is laborious and time-consuming, perhaps taking up to 6 weeks for isolation and definite identification. The

Received 20 Dec. 2001; accepted 27 Jan. 2002.

Corresponding author: Professor D. Taylor-Robinson (e-mail: dtr@vache99.freeserve.co.uk). use of liquid media, particularly if prolific growth were attainable, would provide the opportunity to selectively enhance the growth of Mobiluncus spp. In turn, this would not only enable the dynamics of growth to be determined more easily but also facilitate preparation of antigens for diagnostic and immunological use and the production and examination of diffusible extracellular metabolites, such as the cytotoxin [6] which may be an important pathogenicity factor. It has been suggested recently [7] that $M$. curtisii, in particular, is associated with $\mathrm{BV}$ and is of possible importance in its aetiology. This gave further impetus to determining the efficiency of various liquid media to support growth of Mobiluncus spp., possibly as a prelude to pathogenicity studies, and was the purpose of the current investigation.

\section{Materials and methods}

Bacterial strains and culture media

Three strains each of $M$. curtisii and of M. mulieris isolated from the vagina of women with $\mathrm{BV}$ were received from four laboratories in the UK and Sweden 
[6]. They were subcultured every 5-7 days on Columbia Blood Agar (CBA) (Difco) and incubated anaerobically at $37^{\circ} \mathrm{C}$. Nine media were evaluated: Schaedler broth (BBL), Columbia Blood Broth (CBB; Oxoid), Peptone Starch Dextrose (PSD) broth (Oxoid), Helicobacter pylori medium (Oxoid), fresh tissueculture medium (FTCM) (Flow), spent tissue-culture medium (STCM) (Flow), Brucella broth (Oxoid), Brain-Heart Infusion (BHI) broth (Oxoid) and Cooked Meat Carbohydrate (CMC) medium (Southern Group Laboratories). In addition, in attempts to enhance growth, several agents were added as single supplements to BHI broth as follows: arginine-free base (Sigma) $0.3 \%$, sodium hippurate (Sigma) $1.0 \%$, sodium salts of formate and fumarate $0.3 \%$, and bovine serum $2.5 \%$. Also, heat-inactivated horse serum $10 \%$ and Helicobacter growth supplement (Oxoid) were added as single supplements to PSD broth and to Brucella broth, respectively.

\section{Pre-reduction and inoculation of media}

Agar and broth media were placed in an anaerobic cabinet (Forma Scientific) for $24-48 \mathrm{~h}$ before use to allow for displacement of oxygen. For each of the media tested, a 3-ml sample was inoculated in triplicate with a bacterial strain, as described below. An uninoculated 3-ml sample was kept as a negative control. A suspension of each of the bacterial strains (c. $10^{5}-10^{6} \mathrm{cfu} / \mathrm{ml}$ ) was prepared for each medium by harvesting the growth occurring on a single CBA agar plate into $2 \mathrm{ml}$ of BHI broth. Each 3-ml sample of medium was inoculated with $0.1 \mathrm{ml}$ of the bacterial suspension and incubated under anaerobic conditions $\left(\mathrm{N}_{2} 80 \%, \mathrm{H}_{2} 10 \%, \mathrm{CO}_{2} 10 \%\right)$ at $37^{\circ} \mathrm{C}$ for up to 7 days.

\section{Quantification of Mobiluncus spp. in broth culture}

A 'micromethod' was used [8] that allowed the simultaneous dilution of up to 12 broth cultures in 96-well plates $(12 \times 8$ rows $)$ with a multichannel pipette. Serial 10 -fold dilutions were made by transferring $20-\mu \mathrm{l}$ volumes into successive wells each containing $180 \mu \mathrm{l}$ of BHI broth, up to a dilution of $10^{8}$. Then $10-\mu 1$ samples of each dilution were pipetted in triplicate on to pre-reduced CBA. The plates were incubated anaerobically at $37^{\circ} \mathrm{C}$ for 5 days, after which the small Mobiluncus colonies ( $<1 \mathrm{~mm}$ diameter) were counted with the aid of an electronic colony counter (Jencons). The number of Mobiluncus organisms $/ \mathrm{ml}$ of broth sample was calculated by a modification [8] of the method developed by Miles et al. [9].

\section{Results}

Growth of M. mulieris and M. curtisii in different media

The multiplication of M. mulieris strain A198 in seven different media is shown in Fig. 1. Multiplication of $\geqslant 10$-fold was recorded in five media (Schaedler broth, CBB, PSD broth, STCM and BHI + arginine-free base), but not in FTCM or BHI broth + formate/fumarate. Multiplication did not occur in $H$. pylori medium or CMC medium (not depicted in Fig. 1). Maximal growth (100-fold increase) was shown in Schaedler broth, but viable organisms persisted longer in $\mathrm{CBB}$ and, in other experiments, maximal growth in CBB was similar to that in Schaedler broth.

The multiplication of M. curtisii strain A98 in the same nine media showed a similar pattern to that described for M. mulieris in Fig. 1, although in this case maximal growth was seen in CBB.

\section{Growth of different strains of $M$. mulieris and $M$. curtisii in $C B B$}

The growth of two strains of M. mulieris (A198 and L1000) and two strains of M. curtisii (A98 and A345) in CBB is shown in Fig. 2. The patterns of growth of M. mulieris strain 114.9 and M. curtisii strain A228 (not shown) were similar to that of $M$. curtisii strain A345. It is clear that there are differences in the growth of strains within and between the two species, the greatest difference ( $>10$-fold in maximal growth) being observed between $M$. curtisii strain A98 and M. mulieris strain L1000, multiplication of the latter being the least of all the six strains.

\section{Growth of different strains of M. mulieris and $M$. curtisii in supplemented media}

None of the putative growth factors (see Materials and

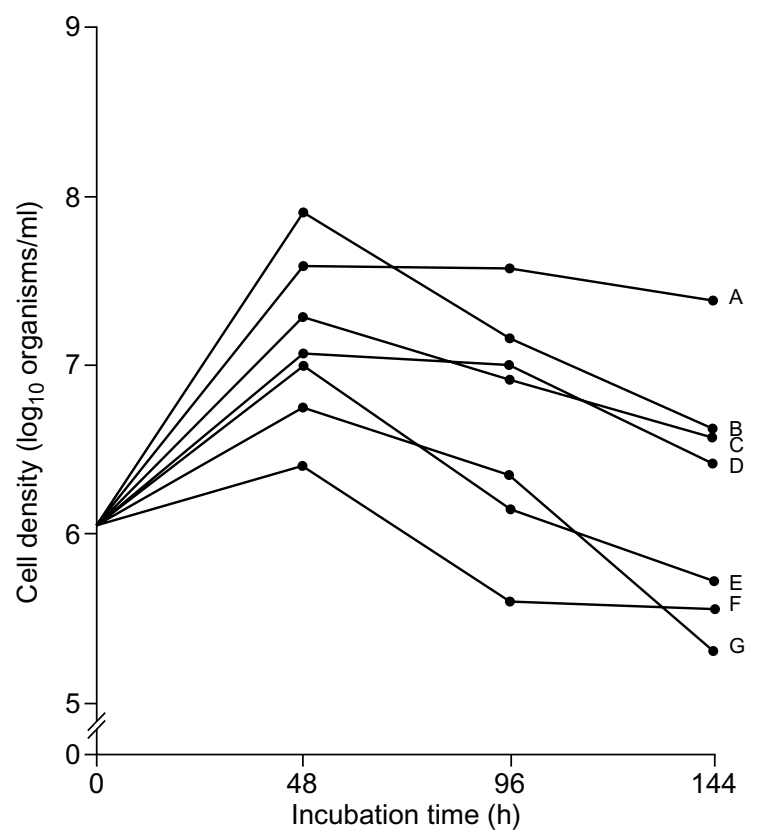

Fig. 1. Growth curves for M. mulieris strain A198 in broth media. A, CBB; B, Schaedler broth; C, PSD broth; D, STCM; E, BHI broth + arginine-free base; F, BHI broth + formate/ fumarate; G, FTCM. 


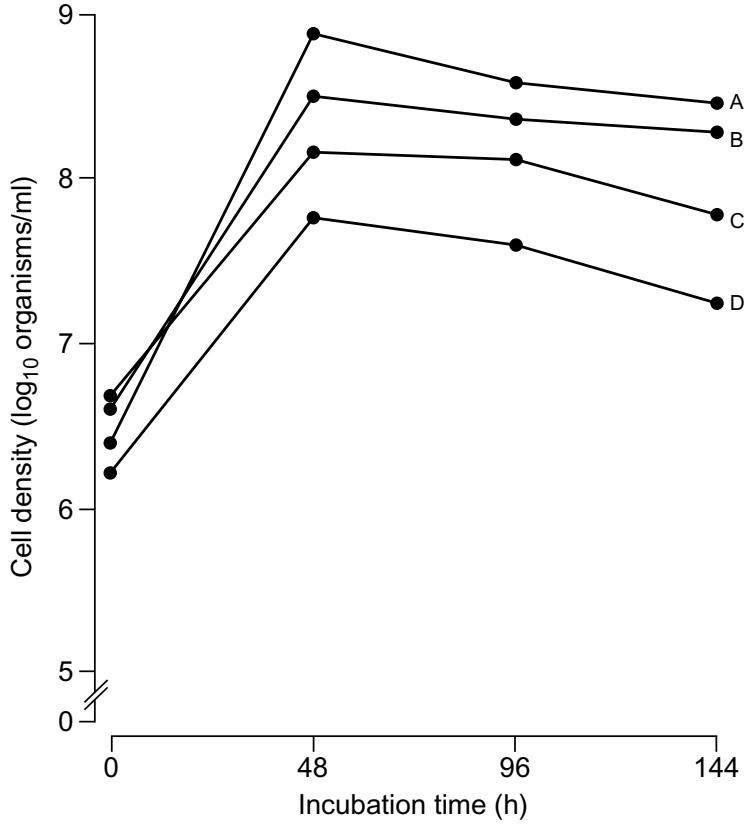

Fig. 2. Growth curves for four strains of Mobiluncus spp. in CBB medium. A, M. curtisii strain A98; C, M. curtisii strain A345; B, M. mulieris strain A198; D, M. mulieris strain L1000.

methods) that were added to BHI broth, with the possible exception of arginine-free base (Fig. 1), had any significant effect on growth of the four strains shown in Fig. 2, or the other two strains mentioned above. However, in PSD medium supplemented with heat-inactivated horse serum $10 \%$ (Fig. 3), after $48 \mathrm{~h}$ there was a 1000 -fold increase in the number of organisms of M. mulieris strain A198 and M. curtisii strain A98, respectively, a titre of $10^{9} \mathrm{cfu} / \mathrm{ml}$ being attained. This was at least 10 -fold more than in

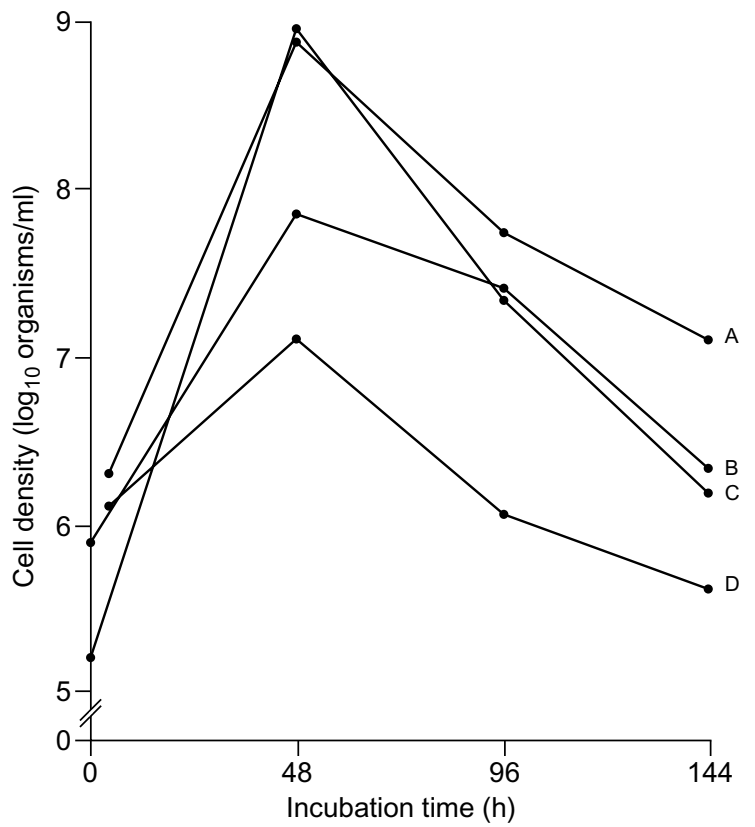

Fig. 3. Growth curves for four strains of Mobiluncus spp. in PSD broth containing heat-inactivated horse serum $10 \%$. A, $M$. mulieris strain A198; B, M. mulieris strain L1000; C, M. curtisii strain A98; D, M. curtisii strain A345. unsupplemented PSD medium. It is noteworthy that, consistent with growth in other media, the growth of strains L1000 and C345 was 10-100-fold less than that of the aforementioned two strains. Furthermore, in the serum-supplemented medium the number of viable organisms of all strains declined rapidly, in comparison with more sustained viability in $\mathrm{CBB}$.

\section{The effect of inoculum size}

In all previous experiments, an inoculum size of $10^{5}-10^{6}$ organisms $/ \mathrm{ml}$ had been used. This had resulted in a titre of $10^{9}$ organisms $/ \mathrm{ml}$ of $M$. mulieris strain A198 and M. curtisii strain A98 in, for example, serum-supplemented PSD medium after $48 \mathrm{~h}$. When the inoculum size was reduced to $(1-2) \times 10^{3}$ organisms $/ \mathrm{ml}$, multiplication in this medium was approximately the same, i.e., 1000 -fold, so that the maximum titre of strain A198 reached only $10^{5}$ organisms $/ \mathrm{ml}$ and of strain A98 $10^{6}$ organisms $/ \mathrm{ml}$ after $144 \mathrm{~h}$.

\section{Discussion}

Mobiluncus spp. are fastidious in their growth requirements and most routine laboratory media either do not contain or have insufficient growth factors to support their multiplication. In the past, the emphasis was on the development of solid media for colony production, thus enabling a distinction to be made between the two species and between strains through colony cloning. For this purpose, providing colony size is acceptable, colony density, which reflects medium sensitivity, is not of over-riding importance. However, the sensitivity of solid medium is important if it is used for primary isolation, where colony development for Mobiluncus spp. may prove difficult. Nevertheless, growth in liquid medium may be even more difficult. Thus, in one study [10] all strains of Mobiluncus spp. tested gave better growth on solid than in liquid medium. This is emphasised in the current study by the fact that most of the liquid media studied were incapable of supporting acceptable levels of growth of either bacterial species, a finding consistent with that in another study [11]. Furthermore, several investigators have shown that even the addition of several putative growth factors did not improve growth in liquid medium [5,10,11], although supplementation with serum proved beneficial, as noted in the current study. The present study also demonstrated that liquid media that supported maximal growth of the Mobiluncus spp. were mainly counterparts of solid media that had been deemed optimal for colony production [5]. Indeed, these observations indicate that investigators in the field need not consider more than a few commercial liquid media, perhaps only one (CBB), for excellent growth. This is based on the fact that the key to high levels of growth in liquid media was found to be: (a) the use of media comprising CBB or PSD plus horse serum $10 \%$, (b) the selection of a bacterial strain 
known to multiply to high titre as some strains, whatever the medium, consistently reached maximal titres greater than attained by others, and (c) an initial inoculum of $10^{5}-10^{6}$ organisms $/ \mathrm{ml}$. Under these circumstances, a viable count of $10^{9}$ organisms $/ \mathrm{ml}$ could be attained consistently in $48 \mathrm{~h}$, maintenance of a high titre being a feature of some media (e.g., CBB), but not others. Such maintenance of viable organisms in liquid medium may be particularly important in the production of soluble metabolic products, such as the cytotoxin. The latter was not demonstrable previously in a centrifuged deposit of organisms or in colonies from solid medium [6].

\section{References}

1. Hillier S, Holmes KK. Bacterial vaginosis. In: Holmes KK, Mårdh P-A, Sparling $\mathrm{PF}$ et al. (eds) Sexually transmitted diseases, 3rd edn. New York, McGraw-Hill. 1999: 563-586.

2. Hjelm E, Hallén A, Forsum U, Wallin J. Anaerobic curved rods in vaginitis. Lancet 1981; 2: 1353-1354.

3. Durieux R, Dublanchet A. Isolement de vibrions anaerobies stricts de la flore vaginale. Rev Inst Lyon 1981; 14: 157.

4. Holst E, Hofmann H, Mårdh P-A. Anaerobic curved rods in genital samples of women. In: Mårdh P-A, Taylor-Robinson D (eds) Bacterial vaginosis. Stockholm, Almqvist \& Wiksell International. 1984: 117-124.

5. Thomason JL, Schreckenberger PC, LeBeau LJ, Wilcoski LM, Spellacy WN. A selective and differential agar for anaerobic comma-shaped bacteria recovered from patients having motile rods and non-specific vaginosis. In: Mårdh P-A, TaylorRobinson D (eds) Bacterial vaginosis. Stockholm, Almqvist \& Wiksell International. 1984: 125-128.

6. Taylor-Robinson AW, Borriello SP, Taylor-Robinson D. Identification and preliminary characterization of a cytotoxin isolated from Mobiluncus spp. Int J Exp Pathol 1993; 74: 357-366.

7. Shwebke JR, Lawing LF. Prevalence of Mobiluncus spp. among women with and without bacterial vaginosis as detected by polymerase chain reaction. Sex Transm Dis 2001; 28 195-199.

8. Borriello SP, Barclay FE. An in-vitro model of colonisation resistance to Clostridium difficile infection. J Med Microbiol 1986; 21: 299-309.

9. Miles AA, Misra SS, Irwin JO. The estimation of the bactericidal power of the blood. J Hyg Camb 1938; 38: $732-749$.

10. Vetere A, Borriello SP, Fontaine E, Reed PJ, Taylor-Robinson D. Characterisation of anaerobic curved rods (Mobiluncus spp.) isolated from the urogenital tract. $J$ Med Microbiol 1987; 23 279-288

11. Sprott MS, Ingham HR, Pattman RS, Clarkson LM, Codd AA, Narang HK. Motile curved bacilli: isolation and investigation. In: Mårdh P-A, Taylor-Robinson D (eds) Bacterial vaginosis Stockholm, Almqvist \& Wiksell International. 1984: 107-111. 